\title{
Downregulation of miR-335 exhibited an oncogenic effect via promoting KDM3A/YAP1 networks in clear cell renal cell carcinoma
}

\author{
Wenqiang Zhang ${ }^{1} \cdot$ Ruiyu Liu ${ }^{1} \cdot$ Lin Zhang $^{1} \cdot$ Chao Wang $^{1} \cdot$ Ziyan Dong $^{1} \cdot$ Jiasheng Feng ${ }^{1} \cdot$ Mayao Luo ${ }^{1} \cdot$

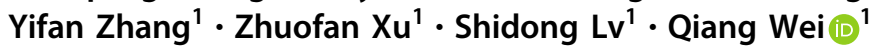

Received: 23 December 2020 / Revised: 3 March 2021 / Accepted: 26 March 2021 / Published online: 23 April 2021

(c) The Author(s) 2021. This article is published with open access

\begin{abstract}
Clear cell renal cell carcinoma (ccRCC) is the most common type of renal cancer affecting many people worldwide. Although the 5-year survival rate is $65 \%$ in localized disease, after metastasis, the survival rate is $<10 \%$. Emerging evidence has shown that microRNAs (miRNAs) play a crucial regulatory role in the progression of ccRCC. Here, we show that miR335, an anti-onco-miRNA, is downregulation in tumor tissue and inhibited ccRCC cell proliferation, invasion, and migration. Our studies further identify the H3K9me1/2 histone demethylase KDM3A as a new miR-335-regulated gene. We show that KDM3A is overexpressed in ccRCC, and its upregulation contributes to the carcinogenesis and metastasis of ccRCC. Moreover, with the overexpression of KDM3A, YAP1 was increased and identified as a direct downstream target of KDM3A. Enrichment of KDM3A demethylase on YAP1 promoter was confirmed by CHIP-qPCR and YAP1 was also found involved in the cell growth and metastasis inhibitory of miR-335. Together, our study establishes a new miR-335/ KDM3A/YAP1 regulation axis, which provided new insight and potential targeting of the metastasized ccRCC.
\end{abstract}

\section{Introduction}

Renal cell cancer (RCC) is one of the most common malignancies worldwide. Based on global cancer 2018 statistics, more than 400,000 patients were newly diagnosed, while more than 170,000 deaths were due to RCC each year [1]. Among all types of RCC, clear cell renal cell carcinoma (ccRCC, also called KIRC) is the most common subtype that accounts for $65-70 \%$ of all renal cancers $[1,2]$. The 5 -year survival rate of localized ccRCC is $65 \%$, however, once the tumor has metastasized, the 5-year survival rate is $<10 \%$ [3]. This occurs because

These authors contributed equally: Wenqiang Zhang, Ruiyu Liu

Supplementary information The online version contains supplementary material available at https://doi.org/10.1038/s41417021-00335-3.

Shidong Lv

Lsd990@163.com

$\triangle$ Qiang Wei

qwei@smu.edu.cn

1 Department of Urology, Nanfang Hospital, Southern Medical University, Guangzhou, Guangdong, China
ccRCC is insensitive to chemotherapy and radiation therapy, and surgery remains the primary choice for patients with ccRCC [4]. Currently, due to the understanding of mechanisms involved in the development and progression of ccRCC, the vascular endothelial growth factor tyrosine kinase inhibitor or rapamycin inhibitor was developed and approved to serve as a first-line treatment for ccRCC [5], highlighting the need to explore ccRCC therapies at the molecular level.

MicroRNAs (miRNAs) are epigenetic regulators of gene expression that contribute to multiple cellular processes, including proliferation, cell fate, and differentiation [5]. MiRNAs regulate gene expression in a sequence-specific manner. Following incorporation into the ribonucleoprotein (RNP) complex RISC (RNA induced silencing complex), miRNAs bind mRNA primarily at their $3^{\prime}$ UTRs and impair the translation and/or stability of the target mRNA [6, 7]. Recently, the functional correlation between miRNAs and cancer has been widely reported. A large number of miRNAs are found to act as onco-miRNAs or anti-oncomiRNAs in human cancer [6, 7]. In ccRCC, several miRNAs are identified with altered expression. miR-28, miR185 , miR-27, and let-7f-2 are upregulated in tumor tissue from patients with ccRCC [8], while miR-141 and miR$200 \mathrm{c}$ are downregulated [9]. Altered expressions of 
miRNAs are related to poor cancer-specific survival after tumor excision. Thus, miRNAs are a suitable prognostic and predictive marker to determine survival of ccRCC patients [10]. More importantly, research has shown that these miRNAs contribute to the ccRCC phenotype [11]. In one report, increased expression of miR-28-5p could promote chromosome instability by inhibiting mitotic checkpoint protein Mad2 in VHL inactivated ccRCC [12]. The decreased expression of miR-141 could promote ccRCC proliferation and metastasis by controlling EphA2 [13]. These studies emphasize the importance of miRNAs in ccRCC carcinogenesis. However, due to the complexity of the miRNA regulatory network, pathophysiological functions and downstream targets of miRNAs in ccRCC require further investigation.

In the present study, we identified that miR-335, a miRNA significantly downregulated in ccRCC, inhibits proliferation, migration, and invasion in a ccRCC cell line. Moreover, we found that the $\mathrm{H} 3 \mathrm{~K} 9 \mathrm{me} 1 / 2$ histone demethylase $\mathrm{KDM} 3 \mathrm{~A}$ is a novel miR-335 downstream target. KDM3A is overexpressed in ccRCC and may promote tumor growth and metastasis by activating yes-associated protein 1 (YAP1) expression through epigenetic mechanisms, thus establishing a miR-335/KDM3A/YAP1 regulation axis. Taken together, our study reveals a new miRNAregulated, epigenetic, tumor-promotional pathway in ccRCC, which provides new insight and potential targeting of metastasized ccRCC.

\section{Materials and methods}

\section{Bioinformatics analysis}

The GEO database (https://www.ncbi.nlm.nih.gov/geo/) was used to search and obtain the human ccRCC miRNA expression dataset GSE12105, GSE16441, and GSE37989, and mRNA expression dataset GSE53757. Differential expression of miRNA and mRNA between ccRCC samples and controls were determined using the "limma" package in $\mathrm{R}$ language, with $\mid \log \mathrm{FCl}>1$ and $p<0.05$ as the threshold. A "pheatmap" package in $\mathrm{R}$ language was used to construct a differential miRNA and mRNA expression heatmap. Three microarray differential miRNA datasets were analyzed using Venn maps (http://bioinformatics.psb.ugent. be/webtools/Venn/). starBase (http://starbase.sysu.edu.cn/ index.php) was used to predict downstream mRNA targets of miR-335. The first 45 mRNAs that were significantly upregulated in ccRCC mRNA dataset GSE53757 were included in the analysis. Finally, GEPIA (http://gepia.ca ncer-pku.cn/index.html) database was used to verify target mRNA expression in ccRCC samples.

\section{Patient enrollment and tissue collection}

A total of 62 patients (27 men and 35 women) pathologically diagnosed with ccRCC were selected from Nanfang Hospital between 2018 and 2020. All patients had undergone radical or partial nephrectomy. All tumor tissues were stored in liquid nitrogen. Patients were classified according to the World Health Organization tumor-lymph nodemetastasis classification system. This study followed The Helsinki Declaration and was approved by the Ethics Committee of Nanfang Hospital (NFEC-201910-K2). All patients' clinical information is shown in Table S1.

\section{Cell culture and transfection}

Renal cancer cell lines 786-O, Caki-1, and Caki-2 were purchased from the American Tissue Culture Collection (ATCC). 786-O cells were cultured with RP1640 medium (Hyclone, Beijing, China). Caki-1 and Caki-2 were cultured with McCoy's 5 A medium (Gibco, Waltham, MA, USA). HK2 cells (kidney proximal tubule epithelial cells) were cultured in DMEM/F-12 medium (Hyclone). 293T cells were purchased from the Chinese Academy of Sciences cell bank and cultured with DMEM medium (Hyclone). All cells were cultured in a medium supplemented with $10 \%$ fetal bovine serum (FBS; Hyclone) and 1\% penicillin/ streptomycin (Invitrogen, Waltham, MA, USA) in a 5\% $\mathrm{CO}_{2}$ incubator at $37^{\circ} \mathrm{C}$.

miR-335 mimics (UCAAGAGCAAUAACGAAAAA UGU) and NC-mimics (CUACAAAGCAAUAACGGGGG AUG) were synthesized by RiboBio (Guangzhou, Guangdong, China). Overexpression pcDNA3.1 (+) vector was purchased from Invitrogen. KDM3A shRNA (shKDM3A-1: GGAGAAGAUUUUAGAGAUA; shKDM3A-2: UGUCAA AGGUGUUCGAGAA), YAP1 shRNA (shYAP1: GGUCA GAGAUACUUCUUAA), and negative control scrambleshRNA (shNC: AUUUCGACCAAGGGCAGUA) were purchased from (Sigma, St. Louis, MO, USA). shRNA, overexpression plasmid, and miRNA mimics were all transfected using LIPO2000 reagent.

\section{Antibodies and reagents}

KDM3A (ab91252, Abcam, Cambridge, MA, USA), Ki67 (ab16667, Abcam, Cambridge, MA, USA), E-cadherin (CST, \#3195, Cell Signaling Technology, Danvers, MA, USA), Vimentin (CST, \#5741, Cell Signaling Technology, Danvers, MA, USA), Hippo pathway antibody sampler kit (CST 8579, Cell Signaling Technology, Danvers, MA, USA), including LATS1, MST1, MST2, YAP1, TAZ, and GAPDH (sc-365062, Santa Cruz, CA, USA) were used in this study. H3K9m2 inhibitor Bix01294, UNC0631, Hippo 
pathway inhibitor verteporfin were purchased from Selleck (Houston, TX, USA).

\section{mRNA expression determined using qRT-PCR}

Cells or tissues were lysed using a Trizol kit (Invitrogen). Total RNA was extracted using the RNeasy Mini Kit (Qiagen, Valencia, CA, USA). cDNA was synthesized using the miRNA First Strand cDNA Synthesis (Tailing Reaction) kit (B532453-0020, Sangon, Shanghai, China). cDNA was made from mRNA detection using a reverse transcription kit (RR047A, Takara, Kyoto, Japan). Ultraviolet-visible spectrophotometry (ND-1000, Nanodrop) was used to detect the quality and concentration of RNA. Total RNA (400 ng) was reversed transcribed using the PrimeScript RT Reagent kit (Takara, Dalian, China). PCR was performed using the SYBR $^{\circ}$ Premix Ex TaqT ${ }^{\mathrm{TM}}$ II (Tli RNaseH Plus) kit (Takara, Japan), according to the manufacturer's instructions, using an ABI Prism 7900 Sequence Detection System (Applied Biosystems, Foster City, CA, USA). U6 and GAPDH were used as internal references. Relative expression was calculated using the $2^{-\Delta \Delta \mathrm{Ct}}$ method. Primer sequences (Huada, Wuhan, China) are shown in Table S2.

\section{Protein expression determined using western blot}

Western blotting was performed using ccRCC tissues and 7860 cells according to previously published protocols [14]. GADPH was used as an internal reference, and the gray intensity of each protein band was analyzed using Image $\mathbf{J}$ software.

\section{Dual-luciferase reporter gene assay}

3'UTR-KDM3A-wild type (WT: GGAAAUGAAUUAC AGGCAGCUG) and 3'UTR-KDM3A-mutant (MUT: GGA AAUGAAUUACAGGCGGCCG) were cloned downstream of the luciferase gene at the pGL3-luciferase reporter plasmid (Promega, Madison, WI, USA). The constructed vector was identified via sequencing. Cells were seeded into 96-well plates at $70 \%$ confluence. After $6 \mathrm{~h}$, luciferase reporter plasmids and RNA were transfected. Six replicates were set up for each sample. DNA and transfection reagents were prepared at Firefly: Renilla: transfection reagent $=0.1 \mu \mathrm{g}$ : $0.01 \mu \mathrm{g}: 0.25 \mu \mathrm{l}$. The final RNA concentration was $100 \mathrm{nM}$ for miRNA and transfection reagent $(0.25 \mu \mathrm{L} /$ well $)$. Diluted DNA, RNA, and transfection reagent were incubated at room temperature for $5 \mathrm{~min}$. Diluted DNA or RNA was then mixed with the transfection reagent and incubated at room temperature for $20 \mathrm{~min}$. Culture medium $(50 \mu \mathrm{l} / \mathrm{well})$ was discarded. DNA and RNA transfection mixtures $(25 \mu \mathrm{L}$ each) were added to each sample. After transfection for $6 \mathrm{~h}$, the medium was replaced with a fresh complete medium. After transfection for a total of $48 \mathrm{~h}$, the medium was discarded. Cells were washed with $100 \mu \mathrm{l}$ PBS. The remaining PBS was blotted when the 96-well plate was tilted. Diluted $1 \times$ protein lysis buffer $(50 \mu \mathrm{l})$ was added to each well and shaken for $15 \mathrm{~min}$ at room temperature. The lysed mixture $(10 \mu \mathrm{l})$ was added to each well in a white opaque 96-well microtiter plate. Premixed LARII $(100 \mu \mathrm{l})$ was added. Luciferase intensity was measured after $2 \mathrm{~s}$. Premixed Stop\&Glo Reagent $(100 \mu \mathrm{l})$ was then added to each well and luciferase intensity was measured again after $2 \mathrm{~s}$. Renilla luciferase was used as a reference. The degree of activation of the target reporter gene was compared based on the ratio of the firefly divided by Renilla luciferase.

\section{Chromatin immunoprecipitation (ChIP)}

ChIP assays were performed as described in our previous work [15] using a Pierce Agarose ChIP Kit (Thermo Fisher Scientific). The antibodies used for the ChIP assays were specific for KDM3A (ab91252, Abcam). ChIP-DNA analysis was then performed using qRT-PCR to evaluate the level of YAP1 at the target loci. YAP1 promoter primers are shown in Table S3.

\section{Cell proliferation was determined using the CCK-8 assay}

Cell suspensions $(100 \mu \mathrm{l})$ were seeded into 96-well plates and incubated in a $37^{\circ} \mathrm{C}$ incubator for $2-4 \mathrm{~h}$. CCK-8 (100 $\mu$ l, Glpbio, Montclair, CA, USA) was added and then incubated for $1-4 \mathrm{~h}$. The absorbance was measured at $450 \mathrm{~nm}$ with a reference wavelength of $600-650 \mathrm{~nm}$.

\section{Cell migration was determined using the scratch test}

After cell transfection at $37{ }^{\circ} \mathrm{C}$ with $5 \% \mathrm{CO}_{2}$ for $24 \mathrm{~h}$, a $10 \mu \mathrm{L}$ pipette tip was used to make one scratch and draw lines on the monolayer cells. Cells were gently washed three times with PBS, serum-free medium was added, and incubated at $37{ }^{\circ} \mathrm{C}$ with $5 \% \mathrm{CO}_{2}$. Images of the cells were taken at 0,24 , and $48 \mathrm{~h}$. Images were taken at distances between the cells on both sides of the scratch and were measured in three randomly selected areas of interest. Scratch distance $(\%)=(24 \mathrm{~h}$ or $48 \mathrm{~h}$ scratch distance $/ 0 \mathrm{~h}$ scratch distance).

\section{Cell invasion was determined using the transwell assay}

Transwell chambers ( $8 \mathrm{~mm}$ pore size; Corning, New York, USA) were used to perform in vitro cell invasion assays in 24-well plates. In a transwell chamber with a polycarbonate 
A

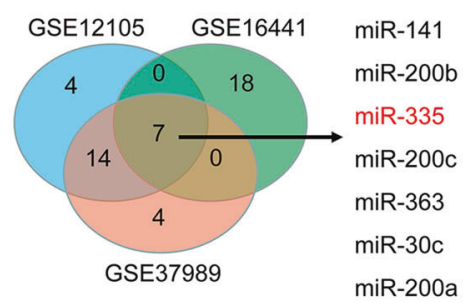

C

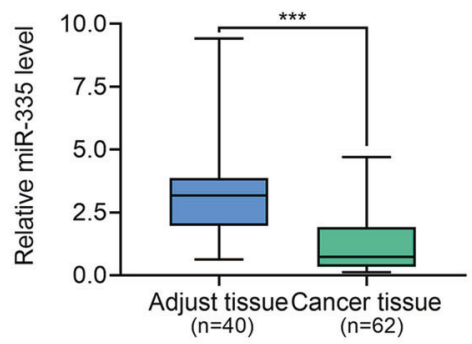

D

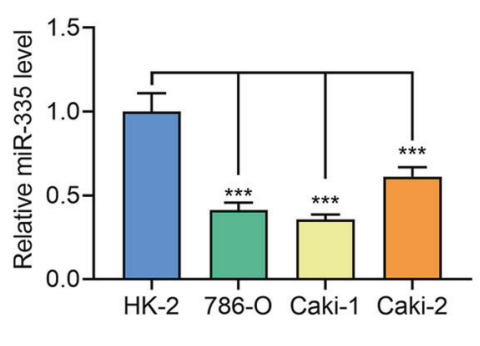

B

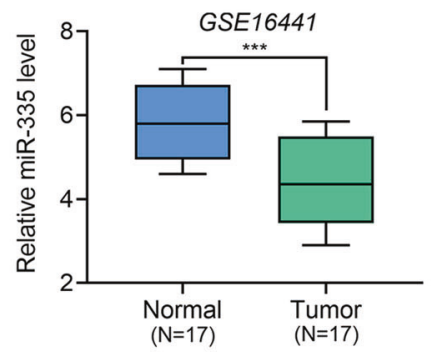

$E$
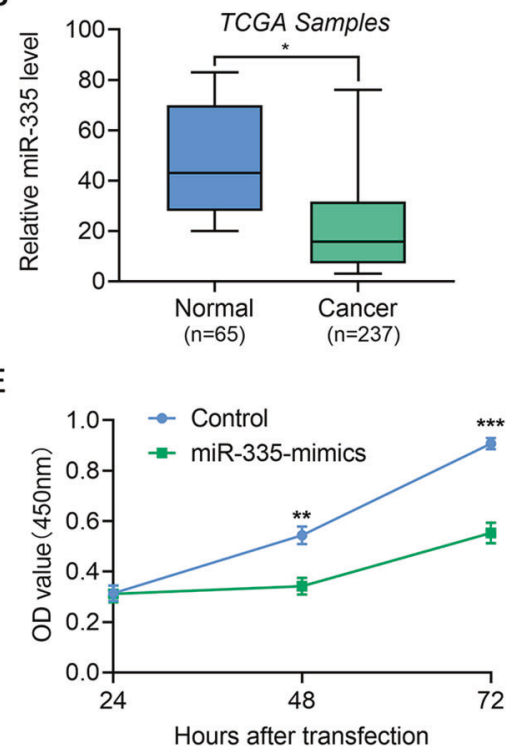

$\mathrm{F}$

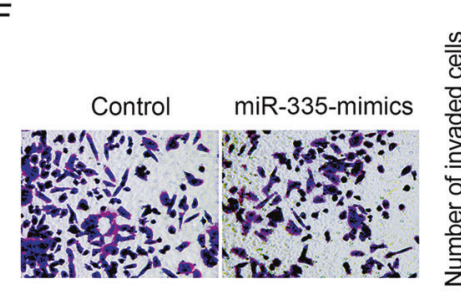

$\mathrm{H}$

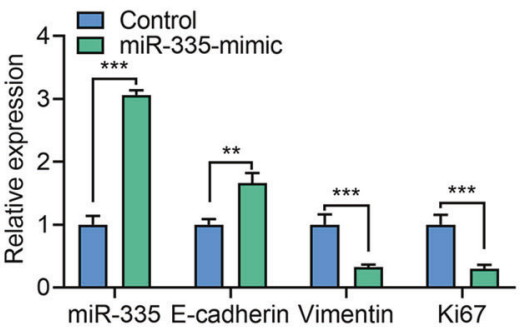

Fig. 1 miR-335 is downregulated in ccRCC and inhibited tumor growth and metastatic capacity. A Venn analysis on the first seven downregulated miRNAs in three datasets, and miR-335 expression in ccRCC in dataset GSE16441. B miR-335 expression in ccRCC in TGCA. C miR-335 expression in ccRCC cancer-adjacent normal tissues and cancer tissues. D miR-335 expression in ccRCC cell lines (HK2 cells were used as normal control). E 786-O cell proliferation was determined using the CCK-8 assay after transfection with miR335 mimics. F 786-O cell invasion was determined using the transwell

membrane containing matrigel, $600 \mathrm{~mL}$ of $20 \%$ FBS DMEM medium was pre-added in the lower chamber and equilibrated at $37^{\circ} \mathrm{C}$ for $1 \mathrm{~h}$. Cells were resuspended in DMEM medium without FBS $48 \mathrm{~h}$ after transfection, inoculated at $1 \times 10^{6}$ cells $/ \mathrm{mL}$ into the upper chamber, and incubated at $37^{\circ} \mathrm{C}$ with $5 \% \mathrm{CO}_{2}$ for $24 \mathrm{~h}$. The transwell chamber was removed and washed twice with PBS. Cells
G
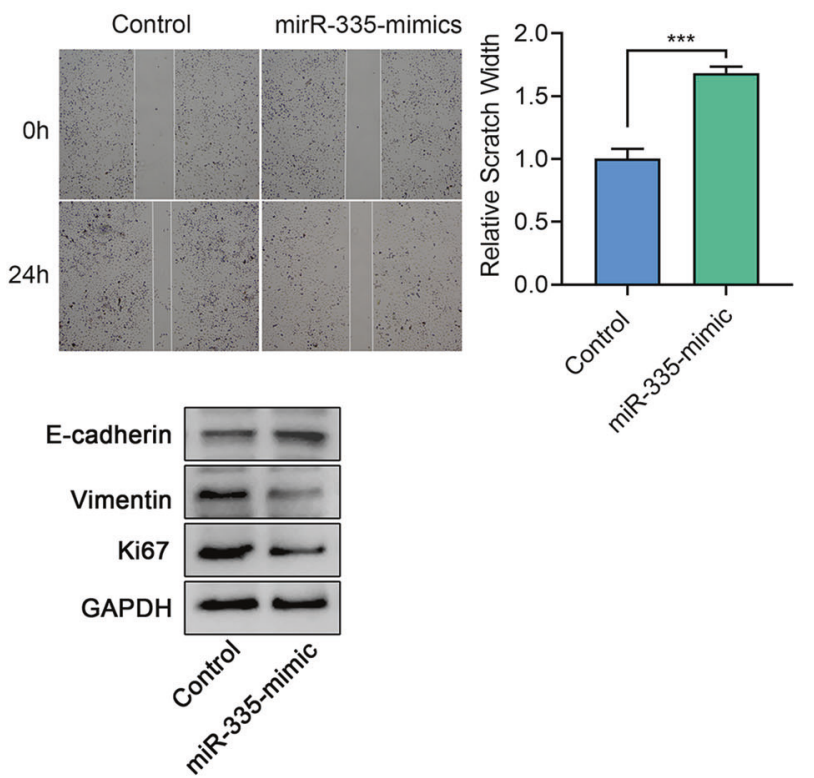

assay after transfection with miR-335 mimics (200×). G 786-O cell migration was determined using the scratch test after transfection with miR-335 mimics. H, I Ki67, E-cadherin and Vimentin mRNA and protein expression in 786-O cells after transfection with miR-335 mimics. Data are expressed as mean \pm standard deviation. Data comparison between two groups was performed using the paired or unpaired $t$ test, where appropriate. Data comparison among multiple groups was performed using one-way ANOVA and Tukey's post hoc test.

were fixed with glutaraldehyde and stained with $0.1 \%$ crystal violet at $4{ }^{\circ} \mathrm{C}$ for $5 \mathrm{~min}$. Cells were then rinsed with PBS twice, surface cells were wiped with a cotton ball and observed under an inverted fluorescence microscope (TE2000, Nikon, Tokyo, Japan). Images were taken in five randomly selected fields. The number of cells passing through the chamber was counted and averaged. 
A

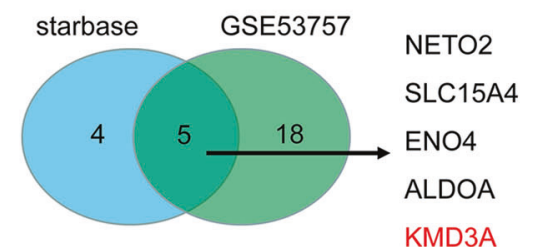

KDM3A Target: 5' gcuggagaaaaaccgaCUCUUGg $3^{\prime}$ hsa-miR-335-5p miR: 3' uguaaaaagcaauaacGAGAACu $5^{\prime}$
B

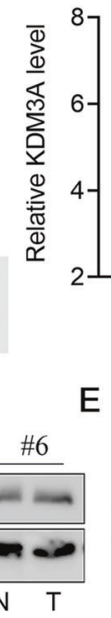

$\mathrm{C}$

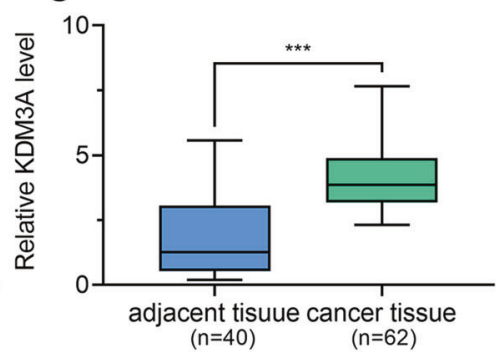

D

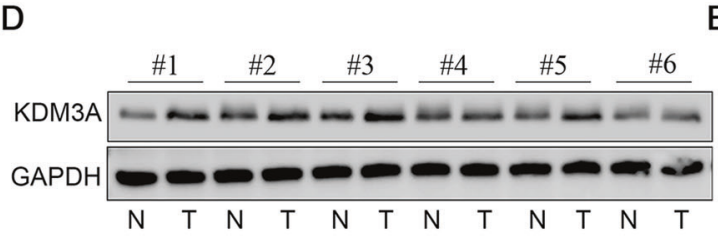

$\mathrm{F}$

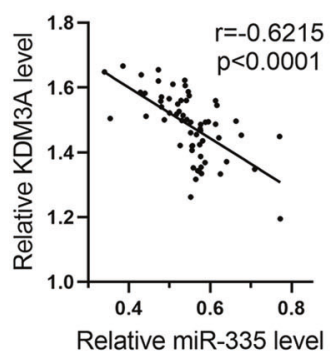

G

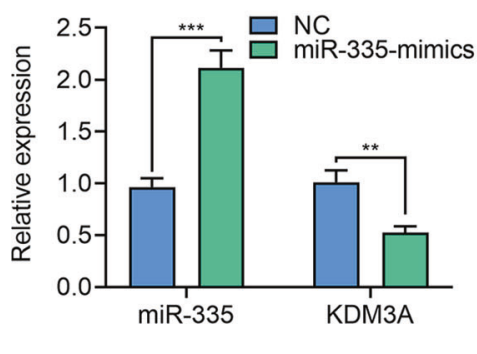

Fig. $2 \mathrm{KDM} 3 \mathrm{~A}$ is a target gene of $\mathrm{miR}-335$ and is overexpressed in ccRCC tumor tissues. A Downstream target genes of miR-335 predicted by starBase and GSE53757 dataset. B Analysis of KDM3A mRNA expression in ccRCC via TGCA samples. C-E KDM3A mRNA and protein expression in ccRCC cancer-adjacent normal tissues and cancer tissues determined using qPCR, western blot, and immunohistochemistry $(200 \times)$. F Correlation between miR-335 and KDM3A mRNA expression in ccRCC cancer tissues. G, H KDM3A

\section{Immunohistochemistry}

Cancer tissues from different groups were fixed in $4 \%$ paraformaldehyde, dehydrated using an alcohol gradient, and embedded in paraffin. Tissues were cut to $4 \mu \mathrm{m}$ serial sections, dewaxed, and rehydrated. Antigen retrieval was performed by microwave heating to remove endogenous peroxidase. After blocking with $10 \%$ goat serum for $30 \mathrm{~min}$ at room temperature, the primary antibody $(50-100 \mu \mathrm{l})$ was added and incubated at $4{ }^{\circ} \mathrm{C}$ overnight in a wet box. Tissues were then incubated with secondary antibody for $1 \mathrm{~h}$, followed by the addition of 50-100 $\mu$ l ABC-kit/StreptavidinHRP and incubated for $30 \mathrm{~min}$ at room temperature. After washing, 50-100 $\mu \mathrm{l}$ of freshly prepared DAB (Maxim, Fuzhou, China) was added and incubated at room temperature for $\sim 1$ min until a yellow color was observed. The reaction was stopped by immediately washing with water.

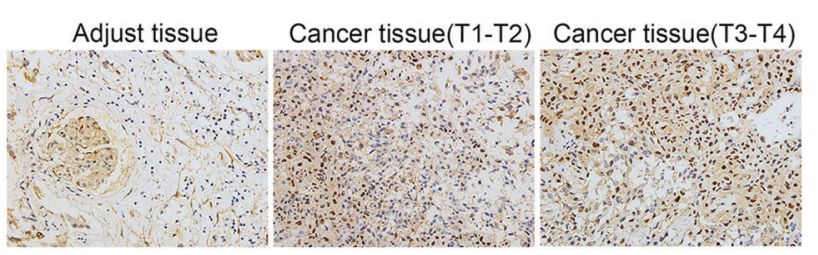

$\mathrm{H}$

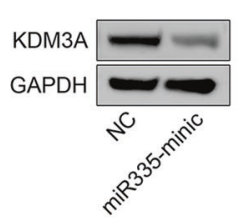

I

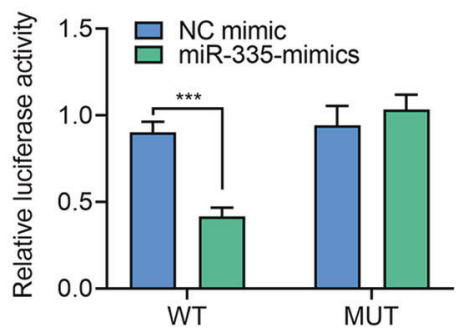

mRNA and protein expression in 786-O cells after transfection with miR-335 mimics. I Binding relationship between miR-355 and KDM3A determined using the dual-luciferase reporter gene assay. Data are expressed as mean \pm standard deviation. Data comparison between two groups was performed using the paired or unpaired $t$ test, where appropriate. Correlation between the two groups was tested using Pearson's correlation.

Tissues were sealed with neutral resin and imaged under a microscope.

\section{Statistical analysis}

All data were analyzed using GraphPad Prism software (GraphPad Software, CA, USA). Data are expressed as mean \pm standard deviation. Data comparison between two groups was performed using the paired or unpaired $t$ test, where appropriate. Data comparison among multiple groups was performed using one-way ANOVA and Tukey's post hoc tests. Data comparison between groups at different time points was performed using repeated measures ANOVA and post hoc Bonferroni tests. Correlation between the two groups was tested using Pearson's correlation coefficient. Calculation data are expressed as rates or percentages and compared using chi-square or Fisher's exact test. $* P<0.05$, 


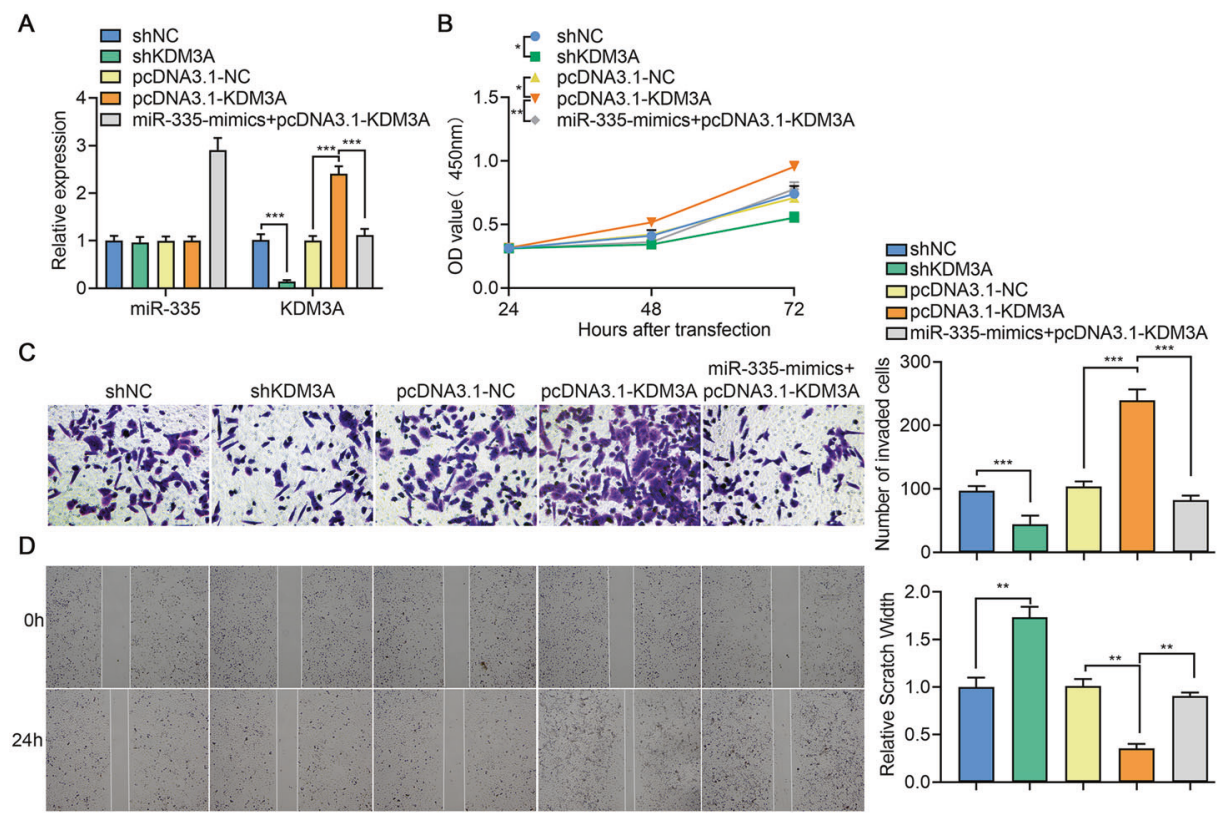

Fig. 3 KDM3A promotes tumor growth and metastasis in ccRCC. A $m i R-335$ and KDM3A mRNA expression tested using qPCR in different groups of 786-O cells. B 786-O cell proliferation determined using the CCK-8 assay in different groups. C 786-O cell invasion determined using the transwell assay in different groups $(200 \times)$. D 786-O cell migration determined using the scratch test in different groups. Data are expressed as mean \pm standard deviation. Data

$* * P<0.01$, and $* * * P<0.001$ were considered statistically significant.

\section{Results}

\section{miR-335 is downregulated in CCRCC and inhibits tumor growth and metastatic capacity}

To explore novel cancer-related miRNAs in ccRCC, we performed a bioinformatics analysis based on three published miRNA microarray datasets accessed from GEO. As shown in Fig. 1A, a total of seven miRNAs were screened out, showing consistently altered expression in all three datasets. Among them, miR-335 displayed the most varied expression, while also being significantly downregulated in TGCA samples (Fig. 1B). This observation was further confirmed in an independent patient cohort (Fig. 1C). Moreover, the expression of miR-335 was dramatically decreased in patients with higher Fuhrman histological grades and $\mathrm{T}$ stages (Table S1). Further, miR-355 levels were markedly lower in primary renal clear cell carcinoma cells 786-O and Caki-2 (However, recent studies have shown that Caki-2 might have the characteristic of papillary comparison between two groups was performed using the paired or unpaired $t$ test, where appropriate. Data comparison among multiple groups was performed using one-way ANOVA and Tukey's post hoc test. Data comparison between groups at different time points was performed using repeated measures ANOVA and Bonferroni post hoc test.

RCC [16]) and cutaneous metastatic renal clear carcinoma cells Caki-1 than that in normal proximal tubular cells (HK2) (Fig. 1D). In the follow-up experiments, we use 786-O cells as the ccRCC model in vitro, for which is a suitable transfection host. These findings suggest that miR-335 may function as an anti-onco-miRNA in ccRCC.

To further investigate the role of miR-335 in ccRCC tumorigenesis, we overexpressed miR-335 using the miRNA mimics in 786-O cells. The CCK-8 assay indicated that miR-335 dramatically blocked the cell growth capacity (Fig. 1E), and the proliferative maker Ki67 downregulated both in mRNA level (Fig. 1H) and protein level (Fig. 1I) in the miR-335 mimic-transfected 786-O cells. Moreover, we also tested whether miR-335 would affect cell invasion and migration capacity using Transwell and wound healing assays. Our results show that both invasion (Fig. 1F) and migration (Fig. 1G) were attenuated in the miR-335 mimictransfected 786-O cells, and the qPCR (Fig. 1H)and western blot results (Fig. 1I) of E-cadherin and Vimentin also show that miR-335 inhibits tumor metastatic capacity. Together, our studies conclude that miR-335 functions as an important anti-onco-microRNA in ccRCC. Downregulation of miR335 inhibits tumor growth and metastasis, which prompts tumor progression. 
Fig. 4 KDM3A up-regulates YAP1 expression through demethylation in 786-O cells. A, B mRNA and protein expression of Hippo pathway molecules after KDM3A knockdown with shRNAs. C Enrichment of KDM3A demethylase on the YAP1 promoter detected using ChIPqPCR. D, E YAP1 mRNA and protein expression after treatment with $\mathrm{H} 3 \mathrm{~K} 9 \mathrm{~m} 2$ inhibitors. F mRNA and protein expression of Hippo pathway molecules after miR-335 and KDM3A overexpression. Data are expressed as mean \pm standard deviation. Data comparison between two groups was performed using paired or unpaired $t$ test, where appropriate. Data comparison among multiple groups was performed using one-way ANOVA and Tukey's post hoc test.
A

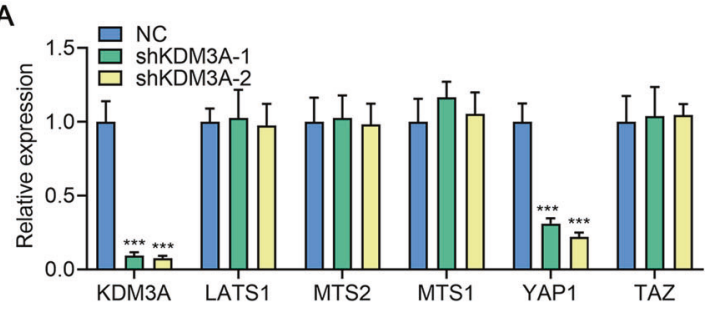

C

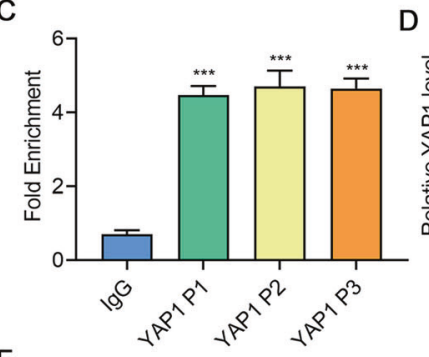

$\mathrm{F}$

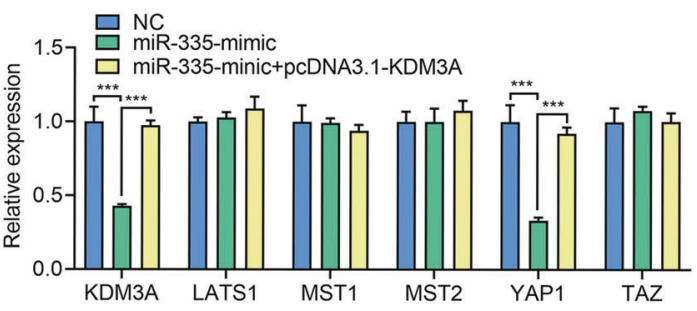

B

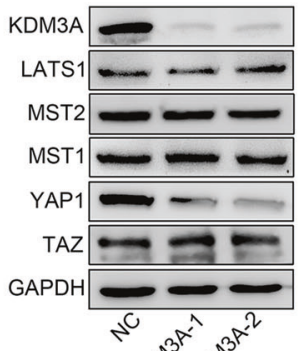

E
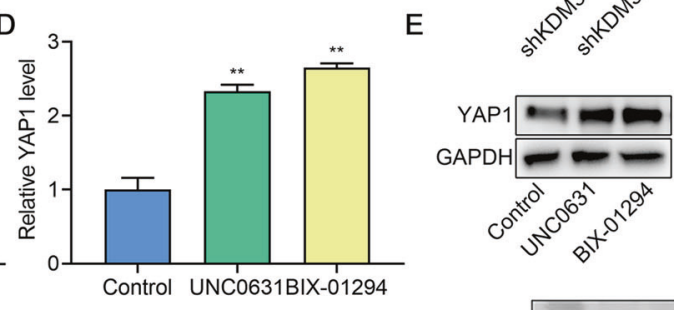

G
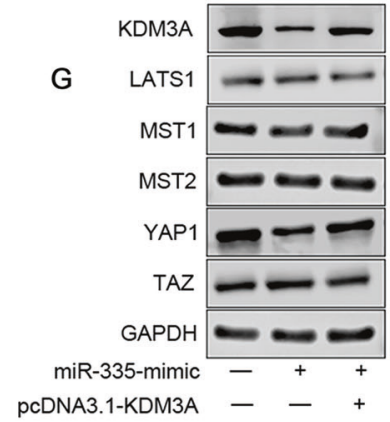

\section{KDM3A is a downstream target of miR-335, and is overexpressed in cCRCC}

The downstream target is very important to understand the pathogenic mechanism of miR-335 in ccRCC. We sought targets associated with ccRCC progression, which showed a negative correlation with miR-335. Therefore, we first predicted the downstream target of ccRCC using starBase and identified 1800 targeted mRNAs. Next, these candidates were explored in a gene array dataset that included 144 paired carcinoma and normal ccRCC tissues. Thereafter, we identified five potential target genes, NETO2, SLC15A4, ENO2, ALDOA, and KDM3A. Among them, histone demethylase KDM3A is an important epigenetic modifier that is reportedly involved in tumor progression in multiple cancers, while miR-335 has a single, highly conserved, predicted target site with the 3-UTR of KDM3A (Fig. 2A). Moreover, we accessed the KIRC TCGA RNAseq dataset and confirmed that the expression of KDM3A was significantly higher in cancer tissues than in canceradjacent normal tissues (Fig. 2B). We observed consistent results in our patient cohort. Both mRNA and protein levels of KDM3A were upregulated in cancer tissues, determined using qPCR (Fig. 2C), western blot (Fig. 2D), and IHC (Fig. 2E). Importantly, a negative correlation was observed between miR-335 and KDM3A in ccRCC tissue samples
(Fig. 2F). Based on these findings, we selected KDM3A as the downstream target of miR-335 for further study.

To verify the regulation between KDM3A and miR-335, we transfected miR-335 mimics into 786-O cells and detected the expression of KDM3A. As shown in Fig. 2G, miR-335 overexpression resulted in a 50\% decrease in KDM3A levels. This downregulation was also observed in KDM3A protein levels (Fig. 2H). In addition, using a dualluciferase reporter system, we confirmed that the KDM3A decrease occurs in response to regulation by miR-335 (Fig. 2I). Overall, our results conclude that KDM3A is a downstream target of miR-335, and that downregulation of miR335 contributes to the induction of KDM3A levels in ccRCC.

\section{KDM3A promotes tumor growth and metastasis in CCRCC}

As a direct downstream target, KDM3A may potentially contribute to cancer growth and metastasis, due to regulation by miR-335. Thus, to explore the function of KDM3A, we knocked down the expression of KDM3A using shRNA in both control 786-O cells and miR-335 mimic-transfected 786-O cells. As expected, miR-335 decreased KDM3A expression, and the loss of KDM3A did not influence miR335 expression (Fig. 3A). The CCK-8 assay demonstrated 
A

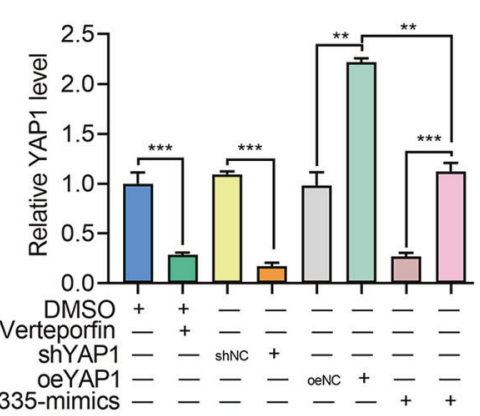

B

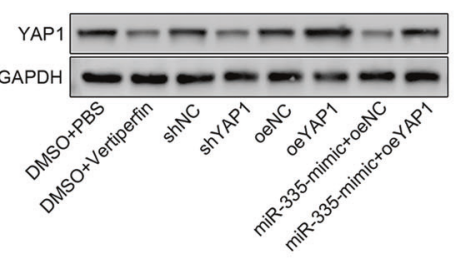

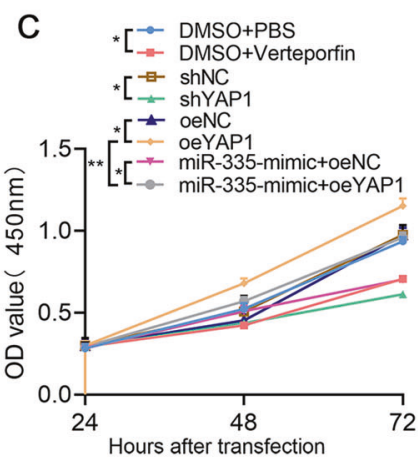

D

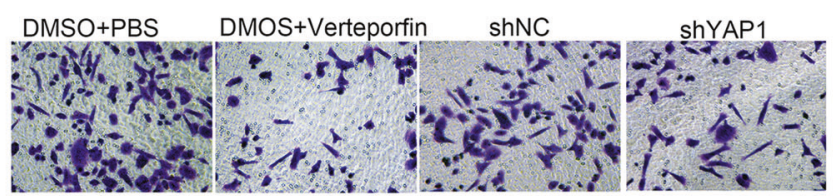

miR-335-mimics

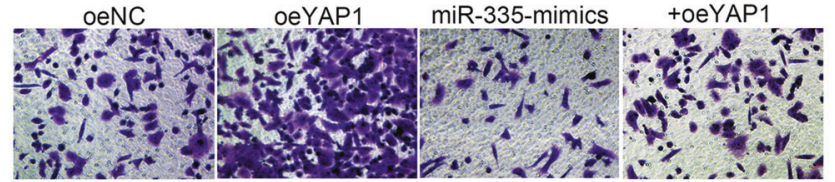

E

DMSO+PBS DMSO+Verteporfin ShNC ShYAP1
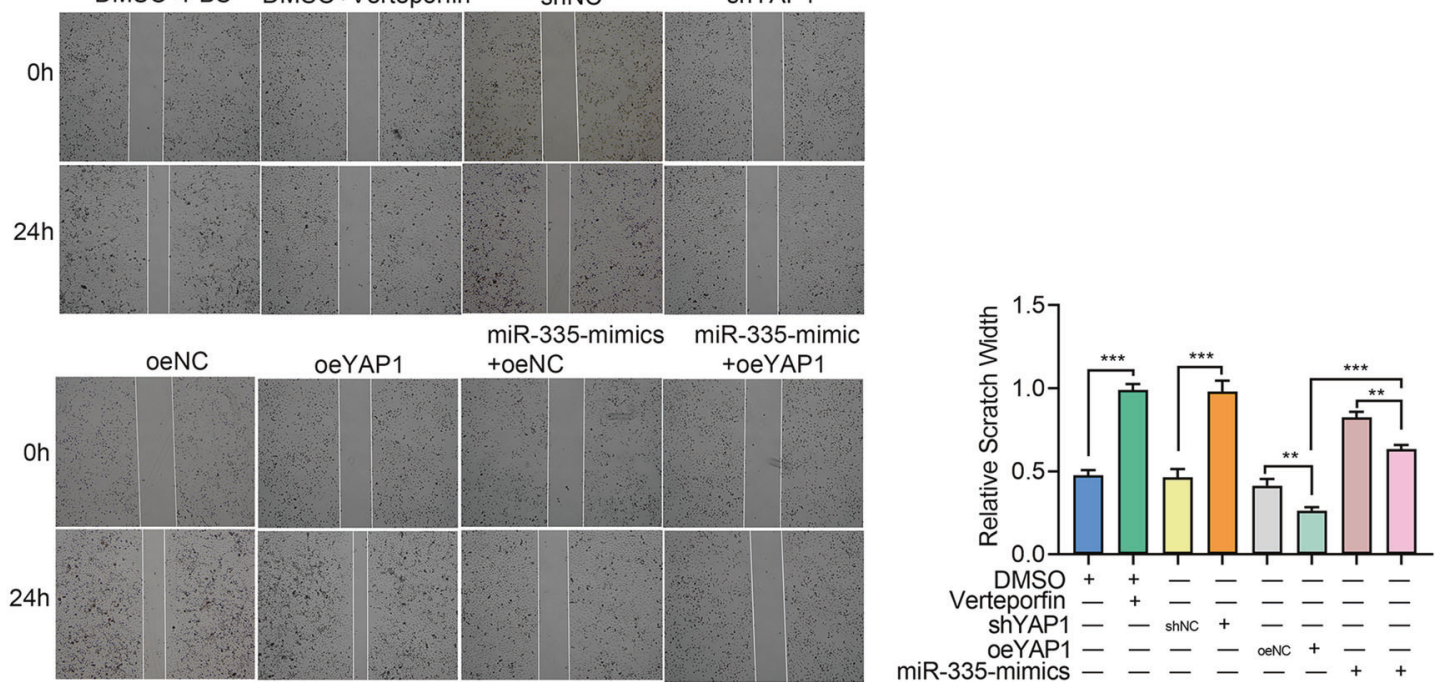

Fig. 5 miR-335 inhibits 786-O cell proliferation, migration, and invasion via the KDM3A/YAP1 axis. A, B YAP1 mRNA and protein expression in 786-O cells with various transfections. E 786-O cell proliferation determined using the CCK-8 assay. F 786-O cell invasion determined using the transwell assay $(200 \times)$. G $786-O$ cell migration determined using the scratch test. Data are expressed as mean \pm standard deviation. Data comparison among multiple groups was performed using one-way ANOVA and Tukey's post hoc test. Data comparison between groups at different time points was performed using repeated measures ANOVA and Bonferroni post hoc test. that cell proliferation was significantly reduced in KDM3A shRNA-transfected cells, while increased in KDM3A overexpressed cells. In line with our previous results, miR335 could inhibit cell proliferation in 786-O cells, and the inhibitory effect of miR-335 was reversed after KDM3A knockdown (Fig. 3B).

In addition, we investigated whether KDM3A contributed to ccRCC metastasis. As shown in Fig. 2C, D, our results showed that a KDM3A deficiency may dramatically attenuate both invasion and migration of ccRCC cells, which was also found in miR-335 overexpressed cells. Overexpression of KDM3A could promote cell invasion and migration. In line with proliferation, the effect of miR335 or KDM3A was reversed when simultaneously cotransfected with miR-335 mimics and KDM3A overexpression plasmids. Taken together, these findings indicate 
Fig. 6 Schematic diagram showing the involvement of miR-335 in ccRCC. miR-355 is under-expressed in ccRCC. Increased KDM3A expression promotes the demethylation of the YAP1 promoter. Increased YAP1 expression (in the Hippo pathway) promotes ccRCC cell proliferation, migration, and invasion.

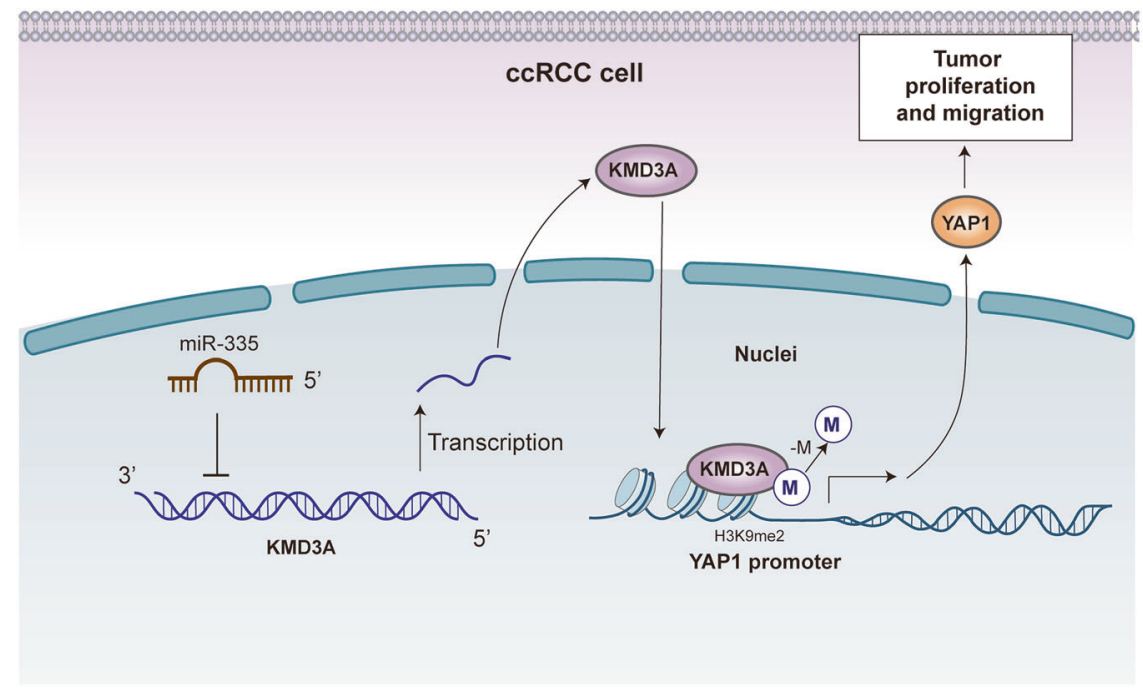

that $\mathrm{KDM} 3 \mathrm{~A}$ is an important oncogene in ccRCC, and promotes tumor growth and metastasis while functioning as the main executor of miR-335.

\section{KDM3A epigenetically activates YAP1 expression}

KDM3A is an $\mathrm{H} 3 \mathrm{~K} 9 \mathrm{me} 1 / 2$ histone demethylase. Due to the methylation of $\mathrm{H} 3 \mathrm{~K} 9$ in promoter regions, which inhibits transcription, KDM3A demethylase activity tends to enhance gene expression. YAP1 is a transcriptional regulator shown to promote tumorigenesis in multiple cancers [17]. In ccRCC, YAP inhibition reportedly impairs LIFRsilencing promotion of cell migration and invasion [18]. Importantly, KDM3A has been shown to upregulate YAP1 and the Hippo pathway in colorectal cancer [19]. Thus, to further address the mechanisms by which KDM3A promotes the oncogenic phenotype in ccRCC, we first investigated gene alteration in the Hippo pathway after KDM3A knockdown in 786-O cells. Results show that KDM3A shRNA transfection reduced the mRNA expression of YAPI (Fig. 4A). This observation was confirmed at the protein level using western blot (Fig. 4B), suggesting that YAP1 is a downstream target of KDM3A. In addition, the direct interaction between KDM3A and YAP1 was identified using ChIP-PCR (Fig. 4C). We identified the robust binding of KDM3A to the YAP1 promoter. Moreover, using histone methyltransferase inhibitors, BIX-01294, and UNC0631, we observed significant YAP1 increases at both mRNA (Fig. 4D) and protein (Fig. 4E) levels. Except KDM3A, the expression of YAP1 may be regulated by miR-335 and reversed after KDM3A overexpression, as observed using qPCR (Fig. 4F) and western blot (Fig. 4G), suggesting a closed relationship between miR-335, KDM3A, and YAP1. Overall, our findings conclude that YAP1 is the direct target of $\mathrm{KDM} 3 \mathrm{~A}$ in $\mathrm{ccRCC}$, and $\mathrm{KDM} 3 \mathrm{~A}$ may regulate YAP1 expression.

\section{The miR-335/KDM3A/YAP1 axis is the key regulator of tumor growth and metastasis in cCRCC}

Our studies have established a regulatory axis including miR-335, KDM3A, and YAP1. Thus, we performed a cotransfection assay to further explore whether YAP1 is also involved in miR-335-induced inhibition of tumor growth and metastasis, as is KDM3A. First, the specific inhibitor, shRNA, and overexpression plasmids were treated or transfected into 786-O cells, respectively. Then, the CCK-8 assay revealed that the inhibitor and shRNA suppressed proliferation, whereas overexpression plasmids promoted 786-O cell proliferation (Fig. 5C). Importantly, after simultaneously co-transfecting YAP1 and miR-335 into 786-O cells, the molecular effects were reversed by opposing treatments. In addition, invasion (Fig. 5D) and migration (Fig. 5E) abilities were also inhibited by the YAP1 inhibitor or shRNA, and promoted by YAP1 overexpression, while the co-transfection of YAP1 and miR-335 showed modest alterations, compared to the single transfection group. Together, our findings verified the functional correlation between miR-335 and YAP1, and identified a new miR-335/KDM3A/YAP1 regulatory axis, which plays an important role in tumor growth and metastasis in ccRCC.

\section{Discussion}

Epigenetic regulatory mechanisms, involving DNA methylation, histone modification at the genomic transcriptional level, and the interaction of non-coding RNAs, 
in particular miRNAs, with mRNA at the posttranscriptional level, are important in the regulation of genes and proteins [20]. Alterations in the expression or regulatory function of miRNAs are key factors for pathological processes. To date, over 300 diseases have been correlated with miRNAs, including oncological diseases [21]. miRNAs may regulate all characteristics of tumors, including control of cell proliferation, apoptosis, angiogenesis, tissue invasion, and metastasis. Here, we focused on miR-335, the most downregulated miRNA observed in three different miRNA array datasets of ccRCC. Functional assays revealed that overexpression of miR-335 inhibits cell proliferation, migration, and invasion in ccRCC cell lines. Moreover, we found $\mathrm{H} 3 \mathrm{~K} 9 \mathrm{me} 1 / 2$ histone demethylase KDM3A as a novel downstream target of miR-335, and identified a new miR-335/KDM3A/YAP1 regulatory axis, which appears to play important roles in ccRCC tumor growth and metastasis. Overall, our studies report a new epigenetic-based tumor-promotional pathway in ccRCC and provide a potential target for the treatment of metastasized ccRCC (Fig. 6).

miRNAs are classified as oncogenic miRNAs or antionco-microRNAs (tumor suppressive) based on their stimulatory or inhibitory effects, respectively, on tumor development. The expression of miR-335 has been shown to be aberrant in multiple cancers. Decreased expression of miR-335 was reported in gastric cancer [22], colorectal cancer [23], and non-small cell lung cancer [24]. However, increased expression of miR-335 was found in several hematologic cancers, such as multiple myeloma [25] and acute leukemia [26]. It is believed that miR-335 can be oncogenic or onco-suppressive depending on the type of cancer. Based on our research, miR-335 was downregulated in ccRCC tumor tissue compared with cancer-adjacent normal tissues, and the expression of miR-335 negatively correlated with histological classification and tumor stage of ccRCC. We also observed a tumor-suppressive role in miR335 mimic-transfected cells. Thus, miR-335 represents an important tumor-suppressive mechanism in ccRCC. In line with our results, Wang et al. reported the decreased expression of miR-335 in a relatively small patient cohort. Their results showed that the low expression of miR-335 was associated with lymph node metastasis, larger tumor size, and poor $\mathrm{T}$ stage [27]. Thus, due to its expression profile and tumor suppressor function, miR-335 may also be used as a metastasis predictive marker for ccRCC [28, 29].

In the present study, to identify a direct target of miR335, we combined miRNA-target interaction predictor, starBase [30], and a ccRCC gene array dataset. Among all candidates, KDM3A was found to be upregulated in ccRCC, and negatively correlated with the expression of miR-335. Moreover, the direct interaction between miR-335 and KDM3A was identified using luciferase assays. KDM3A is a histone modifier that catalyzes the demethylation of transcriptionally repressive mono- and dimethylated histone $\mathrm{H} 3$ lysine 9 ( $\mathrm{H} 3 \mathrm{~K} 9 \mathrm{me} 1 / \mathrm{me} 2)$, thereby mediating transcriptional activation. The deregulation of KDM3A has been reported in skin, hair, and cardiovascular diseases, as well as in multiple cancers, including breast, prostate, and colon cancers [31]. The function of KDM3A is differential and complicated in both physiological and pathological processes. Unlike miR-335, KDM3A is frequently upregulated in cancers and elevated KDM3A correlates with poor disease prognosis [32, 33]. Deletion of KDM3A was found to inhibit colony formation [34], cell proliferation [35], migration [36], and invasion [37]. Thus, KDM3A is an important regulator for tumor growth and metastasis. Here, we explored the pathogenesis of KDM3A in ccRCC. Our results showed that knockdown or overexpression of KDM3A may respectively inhibit or promote cell proliferation, invasion, and migration. Thus, we show that KDM3A is not only a direct downstream target of miR335 , but also a compelling drug target due to its regulatory roles in cancer progression.

Hippo signaling exerts a critical role in modulating cell proliferation and has been demonstrated as an important oncogenic pathway that contributes to the progression of cancer [38]. The Hippo signaling pathway is primarily composed of MST1/2, LATS1/2, YAP, and/or its paralog TAZ [39]. Among them, YAP is the transcriptional coactivator protein that participates in the regulation of downstream genes [40]. The overexpression or over activation of YAP is a common phenomenon in cancers, including non-small cell lung cancer [41], hepatocellular cancer [42], colon cancer [43], gastric cancer [44], and ccRCC [45]. In our study, we showed that the expression of YAP may be regulated by KDM3A directly through an epigenetic role. The binding site of KDM3A was identified in the promoter region of YAP1. Moreover, KDM3A knockdown dramatically influences the intracellular level of YAP1, while using a histone methylation inhibitor would upregulate YAP1 expression. Our findings established a miR-335/KDM3A/YAP1 regulatory axis in ccRCC. Our experiments have shown that the inhibitory role of miR-335 in cell proliferation, invasion, and migration relied on both KDM3A and YAP1, supporting the essential role of the miR-335/KDM3A/YAP1 axis in ccRCC tumor growth and metastasis.

In summary, we screened and identified a new pathogenesis-related miRNA, miR-335, and its downstream regulation axis. Our study lays a foundation for further understanding of the molecular mechanisms of ccRCC progression and indicates that miR-335 and KDM3A are important targets to treat metastasized ccRCC. 
Funding This work was mainly supported by the National Natural Science Foundation of China (81872092); China Postdoctoral Science Foundation (2020M682800).

\section{Compliance with ethical standards}

Conflict of interest The authors declare no competing interests.

Publisher's note Springer Nature remains neutral with regard to jurisdictional claims in published maps and institutional affiliations.

Open Access This article is licensed under a Creative Commons Attribution 4.0 International License, which permits use, sharing, adaptation, distribution and reproduction in any medium or format, as long as you give appropriate credit to the original author(s) and the source, provide a link to the Creative Commons license, and indicate if changes were made. The images or other third party material in this article are included in the article's Creative Commons license, unless indicated otherwise in a credit line to the material. If material is not included in the article's Creative Commons license and your intended use is not permitted by statutory regulation or exceeds the permitted use, you will need to obtain permission directly from the copyright holder. To view a copy of this license, visit http://creativecommons. org/licenses/by/4.0/.

\section{References}

1. Erratum: Global cancer statistics 2018. GLOBOCAN estimates of incidence and mortality worldwide for 36 cancers in 185 countries. CA. 2020;70:313.

2. Moch H, Cubilla AL, Humphrey PA, Reuter VE, Ulbright TM. The 2016 WHO Classification of Tumours of the Urinary System and Male Genital Organs-Part A: Renal, Penile, and Testicular Tumours. Eur Urol. 2016;70:93-105.

3. Znaor A, Lortet-Tieulent J, Laversanne M, Jemal A, Bray F. International variations and trends in renal cell carcinoma incidence and mortality. Eur Urol. 2015;67:519-30.

4. Xu Y, Wu G, Zhang J, Li J, Ruan N, Zhang J, et al. TRIM33 overexpression inhibits the progression of clear cell renal cell carcinoma in vivo and in vitro. Biomed Res Int. 2020;2020:8409239.

5. Gill DM, Hahn AW, Hale P, Maughan BL. Overview of current and future first-line systemic therapy for metastatic clear cell renal cell carcinoma. Curr Treat Options Oncol. 2018;19:6.

6. Esquela-Kerscher A, Slack FJ. Oncomirs - microRNAs with a role in cancer. Nat Rev Cancer. 2006;6:259-69.

7. Berindan-Neagoe I, Monroig PC, Pasculli B, Calin GA. MicroRNAome genome: a treasure for cancer diagnosis and therapy. CA Cancer J Clin. 2014;64:311-36.

8. Gottardo F, Liu CG, Ferracin M, Calin GA, Fassan M, Bassi P, et al. Micro-RNA profiling in kidney and bladder cancers. Urol Oncol. 2007;25:387-92.

9. Nakada C, Matsuura K, Tsukamoto Y, Tanigawa M, Yoshimoto T, Narimatsu T, et al. Genome-wide microRNA expression profiling in renal cell carcinoma: significant down-regulation of miR141 and miR-200c. J Pathol. 2008;216:418-27.

10. Ghafouri-Fard S, Shirvani-Farsani Z, Branicki W, Taheri M. MicroRNA signature in renal cell carcinoma. Front Oncol. 2020;10:596359.

11. Braga EA, Fridman MV, Loginov VI, Dmitriev AA, Morozov SG. Molecular mechanisms in clear cell renal cell carcinoma: role of miRNAs and hypermethylated miRNA genes in crucial oncogenic pathways and processes. Front Genet. 2019;10:320.
12. Hell MP, Thoma CR, Fankhauser N, Christinat Y, Weber TC, Krek W. miR-28-5p promotes chromosomal instability in VHLassociated cancers by inhibiting Mad2 translation. Cancer Res. 2014;74:2432-43.

13. Chen X, Wang X, Ruan A, Han W, Zhao Y, Lu X, et al. miR-141 is a key regulator of renal cell carcinoma proliferation and metastasis by controlling EphA2 expression. Clin Cancer Res. 2014;20:2617-30.

14. Lei C, Lv S, Wang H, Liu C, Zhai Q, Wang S, et al. Leukemia inhibitory factor receptor suppresses the metastasis of clear cell renal cell carcinoma through negative regulation of the yesassociated protein. DNA Cell Biol. 2018;37:659-69.

15. Lv S, Ji L, Chen B, Liu S, Lei C, Liu X, et al. Histone methyltransferase KMT2D sustains prostate carcinogenesis and metastasis via epigenetically activating LIFR and KLF4. Oncogene 2018;37:1354-68.

16. Brodaczewska KK, Szczylik C, Fiedorowicz M, Porta C, Czarnecka AM. Choosing the right cell line for renal cell cancer research. Mol Cancer. 2016;15:83.

17. Wang C, Cheng L, Song S, Wu S, Sun G. Gli1 interacts with YAP1 to promote tumorigenesis in esophageal squamous cell carcinoma. J Cell Physiol. 2020;235:8224-35.

18. Wang $X$, Li XD, Fu Z, Zhou Y, Huang X, Jiang X. Long noncoding RNA LINC00473/miR-195-5p promotes glioma progression via YAP1-TEAD1-Hippo signaling. Int $\mathrm{J}$ Oncol. 2020;56:508-21.

19. Wang HY, Long QY, Tang SB, Xiao Q, Gao C, Zhao QY, et al. Histone demethylase KDM3A is required for enhancer activation of hippo target genes in colorectal cancer. Nucleic Acids Res. 2019;47:2349-64.

20. Baylin SB, Jones PA. Epigenetic determinants of cancer. Cold Spring Harb Perspect Biol. 2016;8:a019505.

21. Braga EA, Fridman MV, Loginov VI, Dmitriev AA, Morozov SG. Molecular Mechanisms in clear cell renal cell carcinoma: role of miRNAs and hypermethylated miRNA genes in crucial oncogenic pathways and processes. Front Genet. 2019;10:320.

22. Zare A, Ahadi A, Larki P, Omrani MD, Zali MR, Alamdari NM, et al. The clinical significance of miR-335, miR-124, miR-218 and miR-484 downregulation in gastric cancer. Mol Biol Rep. 2018;45:1587-95

23. Vickers MM, Bar J, Gorn-Hondermann I, Yarom N, Daneshmand M, Hanson JE, et al. Stage-dependent differential expression of microRNAs in colorectal cancer: potential role as markers of metastatic disease. Clin Exp Metastasis. 2012;29:123-32.

24. Liu J, Bian T, Feng J, Qian L, Zhang J, Jiang D, et al. miR-335 inhibited cell proliferation of lung cancer cells by target Tra2 $\beta$. Cancer Sci. 2018;109:289-96.

25. Qi J, Shi LY, Wu Y, Shen XJ, Yuan J, Jin CJ, et al. Epigenetic silencing of miR-335 induces migration by targeting insulin-like growth factor-1 receptor in multiple myeloma. Leuk Lymphoma. 2019;60:3188-98.

26. Chen L, Shi Y, Li J, Yang X, Li R, Zhou X, et al. LncRNA CDKN2B-AS1 contributes to tumorigenesis and chemoresistance in pediatric T-cell acute lymphoblastic leukemia through miR335-3p/TRAF5 axis. Anticancer Drugs. 2020. Online ahead of print.

27. Wang K, Chen X, Zhan Y, Jiang W, Liu X, Wang X, et al. miR335 inhibits the proliferation and invasion of clear cell renal cell carcinoma cells through direct suppression of BCL-W. Tumour Biol. 2015;36:6875-82.

28. Qin S, Shi X, Wang C, Jin P, Ma F. Transcription factor and miRNA interplays can manifest the survival of ccRCC patients. Cancers. 2019;11:1668.

29. Hao JF, Ren KM, Bai JX, Wang SN, Shao B, Cao N, et al. Identification of potential biomarkers for clear cell renal cell 
carcinoma based on microRNA-mRNA pathway relationships. J Cancer Res Ther. 2014;10:C167-77.

30. Li JH, Liu S, Zhou H, Qu LH, Yang JH. starBase v2.0: decoding miRNA-ceRNA, miRNA-ncRNA and protein-RNA interaction networks from large-scale CLIP-Seq data. Nucleic Acids Res. 2014;42:D92-7.

31. Yoo J, Jeon YH, Cho HY, Lee SW, Kim GW, Lee DH, et al. Advances in histone demethylase KDM3A as a cancer therapeutic target. Cancers. 2020;12:1098.

32. Fan L, Peng G, Sahgal N, Fazli L, Gleave M, Zhang Y, et al. Regulation of c-Myc expression by the histone demethylase JMJD1A is essential for prostate cancer cell growth and survival. Oncogene 2016;35:2441-52.

33. Cui X, Piao C, Lv C, Lin X, Zhang Z, Liu X. ZNFX1 anti-sense RNA 1 promotes the tumorigenesis of prostate cancer by regulating c-Myc expression via a regulatory network of competing endogenous RNAs. Cell Mol Life Sci. 2020;77:1135-52.

34. Cho HS, Toyokawa G, Daigo Y, Hayami S, Masuda K, Ikawa N, et al. The JmjC domain-containing histone demethylase KDM3A is a positive regulator of the G1/S transition in cancer cells via transcriptional regulation of the HOXA1 gene. Int $\mathrm{J}$ Cancer. 2012;131:E179-89.

35. Lee HY, Yang EG, Park H. Hypoxia enhances the expression of prostate-specific antigen by modifying the quantity and catalytic activity of Jumonji C domain-containing histone demethylases. Carcinogenesis. 2013;34:2706-15.

36. Ramadoss S, Guo G, Wang CY. Lysine demethylase KDM3A regulates breast cancer cell invasion and apoptosis by targeting histone and the non-histone protein p53. Oncogene. 2017;36:47-59.
37. Sun L, Yuan Y, Chen J, Ma C, Xu Y. Brahma related gene 1 (BRG1) regulates breast cancer cell migration and invasion by activating MUC1 transcription. Biochem Biophys Res Commun. 2019;511:536-43.

38. Han Y. Analysis of the role of the Hippo pathway in cancer. J Transl Med. 2019;17:116.

39. Zhu C, Li L, Zhao B. The regulation and function of YAP transcription co-activator. Acta Biochim Biophys Sin. 2015;47:16-28.

40. Kowalik MA, Saliba C, Pibiri M, Perra A, Ledda-Columbano GM, Sarotto I, et al. Yes-associated protein regulation of adaptive liver enlargement and hepatocellular carcinoma development in mice. Hepatology. 2011;53:2086-96.

41. Chen HY, Yu SL, Ho BC, Su KY, Hsu YC, Chang CS, et al. $\mathrm{R} 331 \mathrm{~W}$ missense mutation of oncogene YAP1 is a germline risk allele for lung adenocarcinoma with medical actionability. J Clin Oncol. 2015;33:2303-10.

42. Han SX, Bai E, Jin GH, He CC, Guo XJ, Wang LJ, et al. Expression and clinical significance of YAP, TAZ, and AREG in hepatocellular carcinoma. J Immunol Res. 2014;2014:261365.

43. Liang K, Zhou G, Zhang Q, Li J, Zhang C. Expression of hippo pathway in colorectal cancer. Saudi J Gastroenterol. 2014;20:188-94.

44. Lam-Himlin DM, Daniels JA, Gayyed MF, Dong J, Maitra A, Pan $\mathrm{D}$, et al. The hippo pathway in human upper gastrointestinal dysplasia and carcinoma: a novel oncogenic pathway. Int J Gastrointest Cancer. 2006;37:103-9.

45. Schütte U, Bisht S, Heukamp LC, Kebschull M, Florin A, Haarmann $\mathrm{J}$, et al. Hippo signaling mediates proliferation, invasiveness, and metastatic potential of clear cell renal cell carcinoma. Transl Oncol. 2014;7:309-21. 